\title{
Development of Superconducting Tuning Quadrupole Corrector (MQT) Prototypes for the LHC
}

\author{
M. Allitt, S. A. Arshad, A. Hobl, A. Ijspeert, M. Karppinen, D. Krischel, J. Mazet, J. Salminen, M. Schillo, R. Senis, \\ and L. Walckiers
}

\begin{abstract}
The main quadrupoles of the Large Hadron Collider (LHC) are connected in families of focusing and defocusing magnets. In order to make tuning corrections in the machine a number of quadrupole corrector magnets (designated MQT) are necessary. These $56 \mathrm{~mm}$ diameter aperture magnets have to be compact, with a maximum length of $395 \mathrm{~mm}$ and a coil radial thickness of 5 to $7.5 \mathrm{~mm}$, while generating a minimum field gradient of $110 \mathrm{~T} / \mathrm{m}$. Two design options have been explored, both using the "counter-winding" system developed at CERN for the fabrication of low cost corrector coils. The first design, with the poles composed of two double-pancake coils, each counter-wound using a single wire, superposed to create 4-layer coils, was developed and built by ACCEL Instruments GmbH. A second design where single coils were counter-wound using a 3-wire ribbon to obtain 6-layer coils was developed at CERN. This paper describes the two designs and reports on the performance of the prototypes during testing.
\end{abstract}

Index Terms-Corrector magnet.

\section{INTRODUCTION}

$\mathbf{T}$ HE LARGE Hadron Collider (LHC) will incorporate 320 MQT tuning quadrupole superconducting corrector magnets. These are to be mounted in main quadrupole (MQ) cold masses, and will operate in superfluid helium at $1.9 \mathrm{~K}$. They need to provide a minimum field gradient of $110 \mathrm{~T} / \mathrm{m}$, at a nominal operating current of $550 \mathrm{~A}$. Space limitations in the MQ cold mass and the fact that MQT is mounted within an iron shield in order to screen it from nearby busbars (which carry currents up to $13 \mathrm{kA}$ ) constrain the length and diameter of the MQT module to approximately $370 \mathrm{~mm}$ and $150 \mathrm{~mm}$, respectively. The module bore has a diameter of $56 \mathrm{~mm}$. For reliability the MQT working point is specified to be at approximately $60 \%$ of the critical current. Two different designs, designated MQTA and MQTB, have been considered, and prototypes of each constructed and tested.

Manuscript received September 24, 2001.

M. Allitt, A. Ijspeert, M. Karppinen, J. Mazet, R. Senis, and L. Walckiers are with CERN LHC Division, 1211 Geneva 23, Switzerland.

S. A. Arshad was with CERN LHC Division. He is now with McKinsey, London, U.K.

A. Hobl, D. Krischel, and M. Schillo are with Accel Instruments GmbH., 51429 Bergisch Gladbach, Germany.

J. Salminen was with CERN LHC Division. He is now with Nokia, Helsinki, Finland.

Publisher Item Identifier S 1051-8223(02)03425-5.

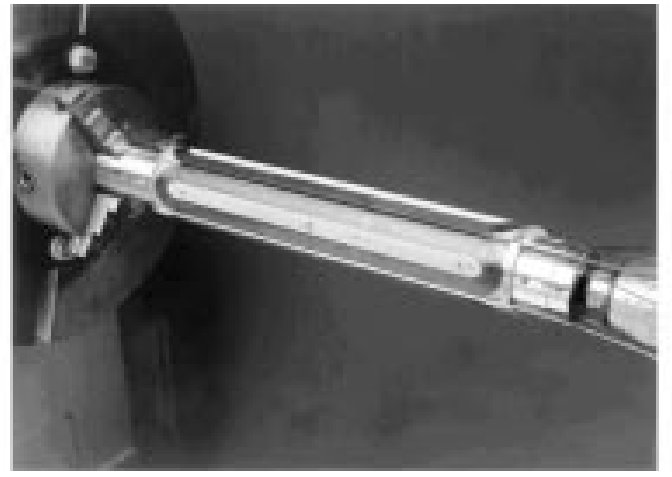

Fig. 1. Assembly of MQTA coils. The coils are doweled to a precisely machined mandrel. A glass fiber plate is mounted at the connection end to support the connections and the assembly glued together using epoxy resin.

\section{Magnet Construction}

The construction methods used for the MQT prototypes have been developed at CERN with the aim of facilitating the industrial production of low cost, robust, superconducting corrector magnets that meet the demanding specifications of the LHC [1]. In both designs, the superconducting coils are wound using enamel insulated, copper stabilized $\mathrm{NbTi}$ conductor of rectangular cross-section, wet-wound around glass-fiber central posts. After curing, these coils are assembled on a mandrel and glued together using epoxy resin (Fig. 1 shows this procedure during the construction of MQTA), with a glass fiber end-plate to provide support for the electrical interconnections. An epoxy pre-preg bandage wrapped around the coils and cured forms an insulation layer, around which iron scissor laminations [2] are stacked to form the return yoke of the magnet.

An aluminum cylinder mounted around the outer diameter of the yoke by shrink fitting holds the complete assembly together and provides the necessary pre-stress, transmitted via the scissor laminations which are free to slide past each other, to the coil assembly to prevent coil movement when the magnet is energized. The amount of pre-stress is controlled by precise machining of the bandage outer diameter, thus defining the radial interference between the yoke outer diameter and the shrinking cylinder inner diameter.

\section{MAGNeTIC DesignS}

In both cases the magnetic designs were optimized using ROXIE [2]. MQTA was also modeled using the 
TABLE I

MQT PROTOTYPES CONDUCTOR PROPERTIES

\begin{tabular}{lll} 
& \multicolumn{1}{c}{ MQTA } & \multicolumn{1}{c}{ MQTB } \\
Insulated dimensions & $1.43 \mathrm{~mm} \times 0.63 \mathrm{~mm}$ & $1.25 \mathrm{~mm} \times 0.73 \mathrm{~mm}$ \\
Insulation thi ckness & $0.06 \mathrm{~mm}$ & $0.06 \mathrm{~mm}$ \\
Cu/Sc ratio & $1.6-1.62$ & 1.6 \\
Filament diameter & $7 \mu \mathrm{m}$ & $7-10 \mu \mathrm{m}$ \\
Residual resistance ratio & $136-142$ & $>100$ \\
Critical current $(5 \mathrm{~T}, 4.2 \mathrm{~K})$ & $695 \mathrm{~A} \perp, 799 \mathrm{~A} / /$ & $\geq 650 \mathrm{~A} \perp \geq 715 \mathrm{~A} / /$ \\
\hline
\end{tabular}

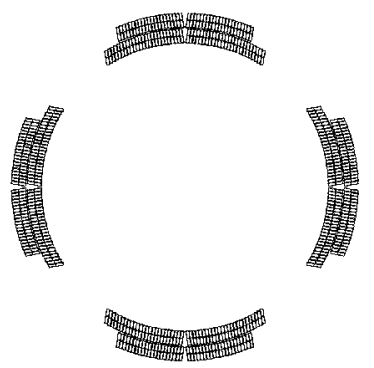

Fig. 2. MQTA coil cross section. Two-layer coils are superposed and connected in series to make four-layer coils.

OPERA/TOSCA ${ }^{{ }_{1}}$ software package to provide a cross-check with the ROXIE results. The goal of the optimization was to configure the coil block geometry so as to simultaneously maximize the integrated quadrupole field component $\mathrm{B}_{2}$ and minimize the $b_{6}$ and $b_{10}$ field harmonics. To keep the complexity of the construction to a minimum in order to obtain designs well suited to inexpensive production of series quantities, the use of spacers within coil blocks was not considered. This results in insufficient free parameters to allow both these harmonics to be reduced to zero at the same time. The optimizations were carried out using three-dimensional (3-D) models since the relatively short length of the coil results in a strong influence from the coil ends, in particular on the $b_{6}$ field harmonic. In both cases 2-D mechanical models were used to calculate the radial interference required between the yoke and the shrinking cylinder at room temperature in order to obtain the necessary pre-stress levels at $1.9 \mathrm{~K}$.

\section{A. MQTA}

The MQTA was developed and built by Accel Instruments $\mathrm{GmbH}$, under contract from CERN. The design approach concentrated on the use of "double-pancake" coils, made by counter-winding a single wire (see Table I) so that each double-pancake contains two layers wound simultaneously. Several winding tests were performed before finalizing the central post design in order to facilitate the coil winding process and maximize the precision of the conductor placement. In the resulting optimized design each pole of the quadrupole is composed of two such double-pancakes superposed and connected in series by soldering to give four-layer coils, so that the magnet in fact consists of 8 coils. The coil cross section is shown in Fig. 2.

The iron yoke increases the field gradient by $60 \%$, and holes in the yoke control the effects of the iron saturation on the field

\footnotetext{
${ }^{1}$ Registered trademark of Vector Fields Ltd., Oxford, England.
}

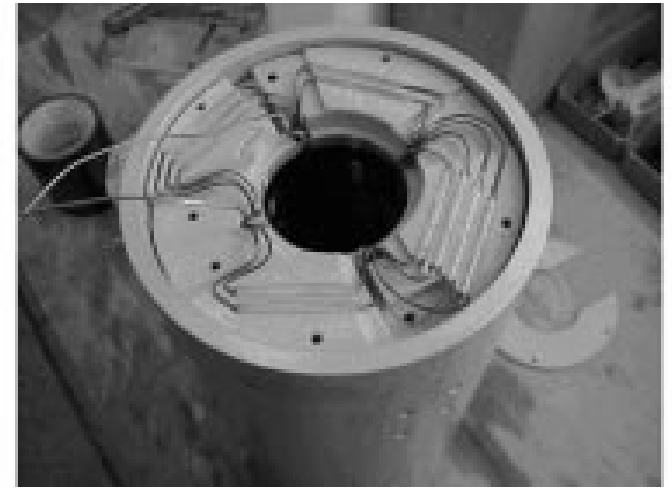

Fig. 3. MQTB electrical connections. Not only must the 4 coils be connected in series, but also the 6 layers in each coil.

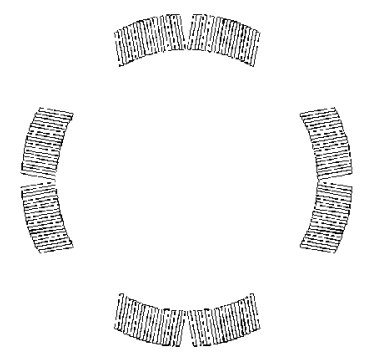

Fig. 4. MQTB coil cross-section. Coils are made by counterwinding a 3-wire ribbon to make 6-layer coils.

harmonics. The dimensions and positions of these holes were included in the optimization procedure. Attempts to control $b_{10}$ by varying the relative angular positions of the inner and outer coil blocks proved unsuccessful.

\section{B. $M Q T B$}

The LHC will contain approximately 6500 corrector magnets with 18 different types of coil. To reduce conductor costs it is desirable to standardize as far as possible the conductors used in these magnets. In order for the MQT conductor to be compatible with other corrector types, a second design, MQTB, was developed at CERN using a standardized wire (see Table I). A four-layer design using this wire cannot provide the minimum required gradient of $110 \mathrm{~T} / \mathrm{m}$ with an adequate safety margin, and also there is considerable interest in increasing the field gradient beyond this minimum, to at least $120 \mathrm{~T} / \mathrm{m}$. Therefore, a six-layer design was developed, with the magnet composed of four coils of six layers, each counter-wound using a ribbon of three wires. This ribbon was manufactured at CERN using a purpose-built machine that uses epoxy resin to glue single wires together and cures the resin in a continuous process. While this manufacturing method obviates the need to make series connections between superposed pancakes, it is instead necessary to make them between the conductors that make up the ribbon (see Fig. 3).

The MQTB coil cross section is shown in Fig. 4. In this design, variation of the $b_{6}$ harmonic with yoke saturation is controlled by optimizing the dimensions of the yoke and the air gap between the yoke and the surrounding iron shield, rather than by the use of holes in the yoke. 
TABLE II

COMPARISON OF MQT PROTOTYPES MAGNETIC DESIGN

\begin{tabular}{|c|c|c|}
\hline & MQTA & MQTB \\
\hline Tums/coil (azimuhal $\times$ radial) & $25 \times 2 ; 21 \times 2$ & $20 \times 6$ \\
\hline Noninal carr ant (A) & 550 & 550 \\
\hline Gradient $(\mathrm{T} / \mathrm{m})$ & 115 & 131 \\
\hline Magnetie length (m) & 0.33 & 0.32 \\
\hline by (units) & $\mathbf{3}$ & 24 \\
\hline$b_{10}$ (areits) & -14 & -15.2 \\
\hline Theoretical quench current of $4.3 \mathrm{~K} / 1.9 \mathrm{~K}(\mathrm{~A})$ & 600,900 & 670965 \\
\hline
\end{tabular}

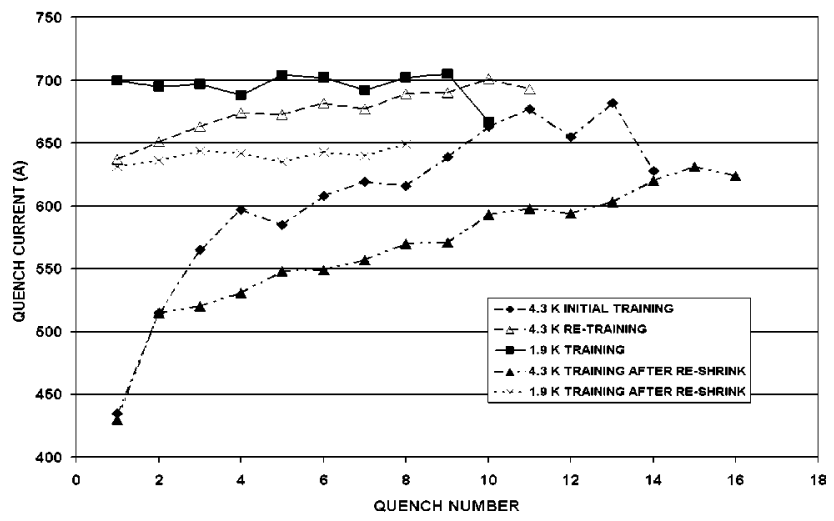

Fig. 5. MQTA training. No increase in the critical current is observed at $1.9 \mathrm{~K}$, indicating a mechanical limitation to the magnets' performance.

The 2-D design values of the magnetic parameters of MQTA and MQTB are summarized in Table II. The relatively high $b_{6}$ is required to cancel the contribution from the coil ends in both designs.

\section{Test Results}

\section{A. Training Tests}

The MQTA was initially trained at Accel Instruments at $4.3 \mathrm{~K}$. Re-training at $4.3 \mathrm{~K}$ and further training at $1.9 \mathrm{~K}$ were then carried out at CERN. The results are shown in Fig. 5. During the initial training at $4.3 \mathrm{~K}$ the nominal operating current was exceeded at the third quench, and the magnet trained steadily to a value approaching the theoretical estimate of the critical current. At the final quench of this test program some de-training was seen. On re-training at CERN, the first quench agreed well with the final training quench at Accel Instruments and once again the magnet trained steadily to about $700 \mathrm{~A}$. On cooling the magnet to $1.9 \mathrm{~K}$ no increase in the critical current was visible. Combined with the slow training at $4.3 \mathrm{~K}$, this implies the presence of some mechanical limitation to the performance of the magnet.

To investigate the possibility that the lack of improvement in critical current at $1.9 \mathrm{~K}$ was due to insufficient pre-stress, the radial interference between the shrinking cylinder and the yoke was increased, changing the pre-stress of the coils at $1.9 \mathrm{~K}$ from an estimated $60 \mathrm{MPa}$ to approximately $80 \mathrm{MPa}$. More training tests were then carried out. It is apparent that the increased pre-stress had a detrimental effect on the performance of the magnet, with the first $4.3 \mathrm{~K}$ quench occurring at $430 \mathrm{~A}$ and being followed by slow training up to about $620 \mathrm{~A}$. Cooling

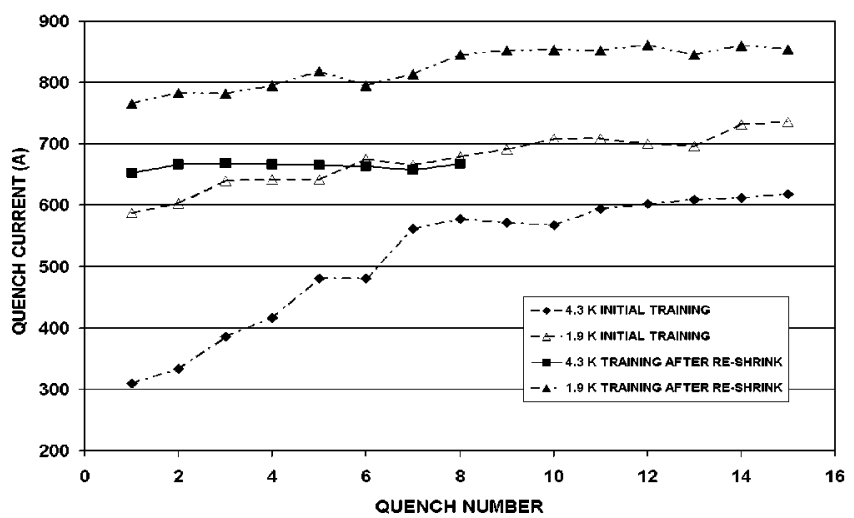

Fig. 6. MQTB training. After increasing the pre-stress the magnet reached the theoretical critical current at both $4.3 \mathrm{~K}$ and $1.9 \mathrm{~K}$.

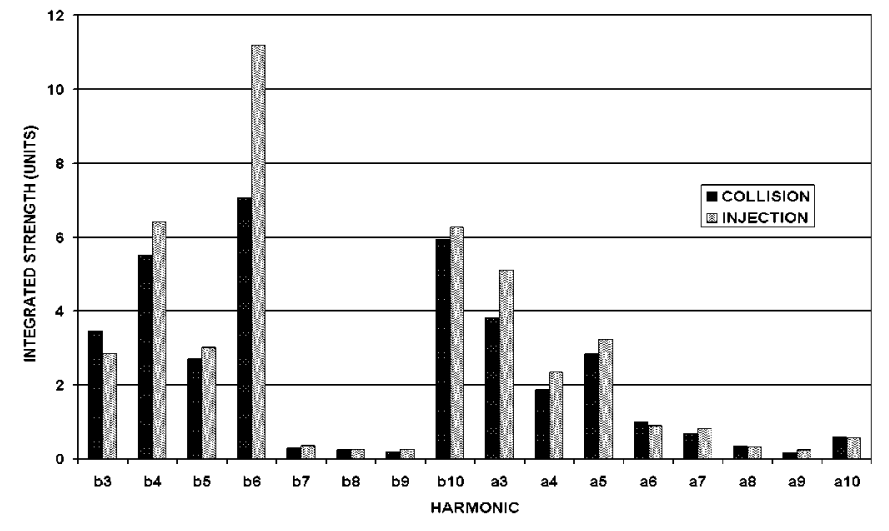

Fig. 7. Magnitudes of the measured integrated field harmonics of MQTA, at a reference radius of $17 \mathrm{~mm}$, in units of $\mathrm{B}_{2} / 10^{4}$.

to $1.9 \mathrm{~K}$ produced only a further $30 \mathrm{~A}$ increase in the critical current.

MQTB underwent training tests at $4.3 \mathrm{~K}$ and $1.9 \mathrm{~K}$ at CERN. In the first series of tests, training was slow and the magnet remained well below the theoretical critical current, as shown in Fig. 6.

To see the effect of increasing the pre-stress in MQTB, a 0.05 $\mathrm{mm}$ stainless steel foil was then fitted between the shrinking cylinder bore and the yoke outer diameter to increase the radial interference to $0.12 \mathrm{~mm}$ and the pre-stress to an estimated 80 $\mathrm{MPa}$ at $1.9 \mathrm{~K}$. The magnet was then re-tested. In this instance this procedure significantly improved the performance of the magnet. At $4.3 \mathrm{~K}$ the magnet began training at $650 \mathrm{~A}$, although it should be noted that it is common for an impregnated magnet to retain memory of previous training tests after the pre-stress has been modified. The theoretical critical current, $670 \mathrm{~A}$, was reached at the second quench. Cooling to $1.9 \mathrm{~K}$ then brought an immediate improvement to over $760 \mathrm{~A}$, following which the magnet trained to a limit of $860 \mathrm{~A}$, again in good agreement with the calculated critical current.

\section{B. Magnetic Field Quality}

The magnetic field was measured as a function of excitation current at CERN at $1.9 \mathrm{~K}$ for both MQTA and MQTB. In the LHC, the collison and injection energies are $7 \mathrm{Tev}$ and $0.45 \mathrm{TeV}$, 


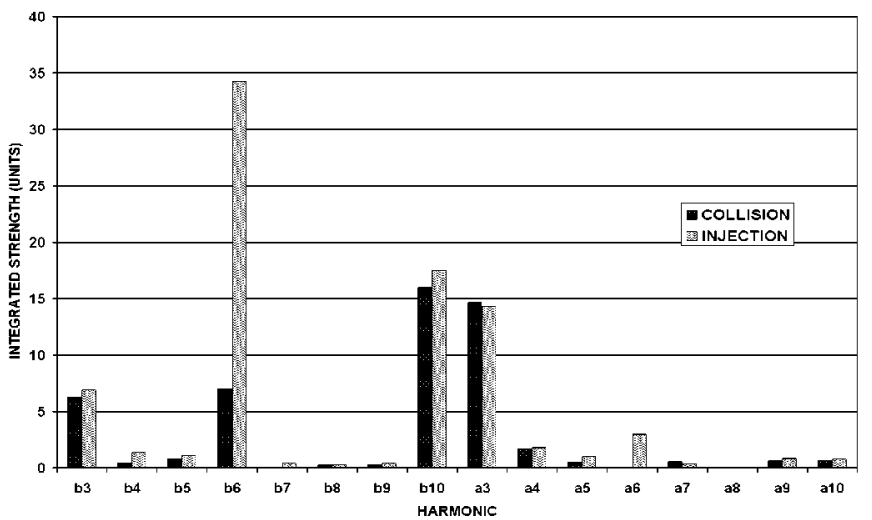

Fig. 8. Magnitudes of the measured integrated field harmonics of MQTB, at a reference radius of $17 \mathrm{~mm}$, in units of $\mathrm{B}_{2} / 10^{4}$.

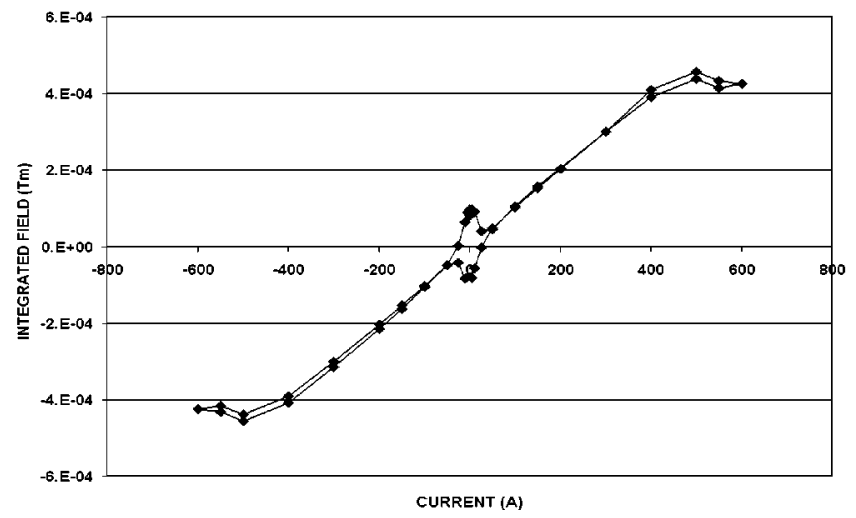

Fig. 9. Integrated $\mathrm{B}_{6}$ harmonic of MQTA, at a reference radius of $17 \mathrm{~mm}$, measured passing through zero current from opposite directions.

respectively. With a target gradient at collision of $110 \mathrm{~T} / \mathrm{m}\left(\mathrm{B}_{2}\right.$ $=1.87 \mathrm{~T}$ at a radius of $17 \mathrm{~mm}$ ) and therefore at injection of 110 $\times 0.45 / 7=7 \mathrm{~T} / \mathrm{m}$, the loadline measurements of MQTA give an excitation of $541.2 \mathrm{~A}$ at collision and $33.9 \mathrm{~A}$ at injection. For MQTB, taking the target gradient at collision to be $120 \mathrm{~T} / \mathrm{m}$ $\left(\mathrm{B}_{2}=2.04 \mathrm{~T}\right.$ at $\left.17 \mathrm{~mm}\right)$, the currents at collision and injection were found to be $493.4 \mathrm{~A}$ and $33.1 \mathrm{~A}$, respectively. The integrated strength of the magnetic field multipoles at collision and injection strengths are shown in Fig. 7 for MQTA and Fig. 8 for MQTB.

Nonlinear effects from iron saturation are evident in both magnets. At collision, the observed magnetic field components of both prototypes were found to lie within the range of variation to be expected, taking into account the random field errors introduced by the use of general tolerances of $\pm 0.1 \mathrm{~mm}$ during their fabrication. MQTB has a higher residual integrated $b_{10}$ than MQTA by about 10 units, $b_{6}$ is similar for the two magnets at approximately seven units.

However, at injection MQTB was found to have a higher than expected $b_{6}$ of 34 units $\left(1.4 \times 10^{-4} \mathrm{Tm}\right)$, compared to MQTA

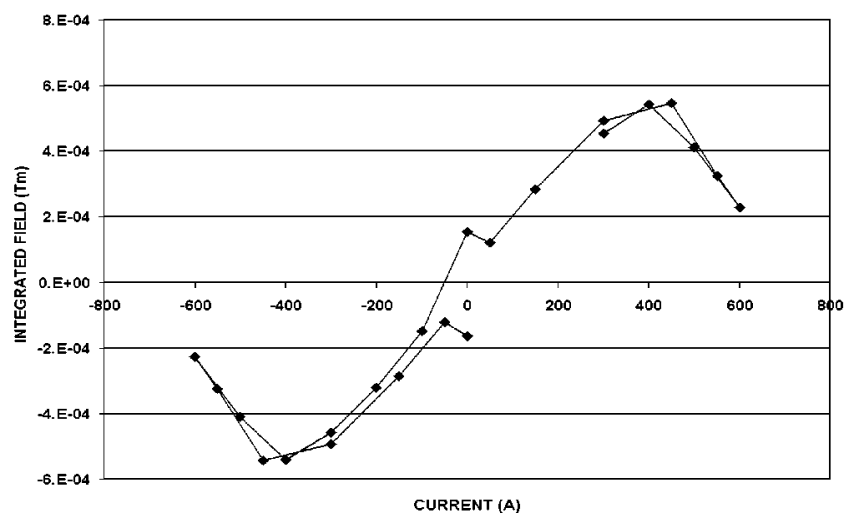

Fig. 10. Integrated $B_{6}$ harmonic of MQTB, at a reference radius of $17 \mathrm{~mm}$, measured passing through zero current from opposite directions.

with a $b_{6}$ of 11 units $\left(4.5 \times 10^{-5} \mathrm{Tm}\right)$. Further investigation of the multipoles by making measurements when passing through zero current from opposite directions (see Figs. 9 and 10), shows that hysteresis due to persistent currents in the superconducting filaments makes a significant contribution at low excitations to the $b_{6}$ harmonics of both magnets.

\section{CONCLUSION}

Two different designs of MQT tuning quadrupole corrector magnet have been developed and tested. The MQTA, with fourlayer coils designed to meet the original $110 \mathrm{~T} / \mathrm{m}$ gradient requirement of the LHC, provided this nominal gradient at $78 \%$ of the loadline at $4.3 \mathrm{~K}$, but the critical current did not improve at $1.9 \mathrm{~K}$. Increasing the pre-stress in the coils caused a deterioration in the performance of the magnet. The MQTB with six-layer coils designed to produce a gradient of $120 \mathrm{~T} / \mathrm{m}$ trained to theoretical critical current at both $4.3 \mathrm{~K}$ and $1.9 \mathrm{~K}$, and provided the nominal gradient at $57 \%(73 \%)$ of the loadline at 1.9 $\mathrm{K}(4.3 \mathrm{~K})$. For both prototypes magnetic field multipoles at collision energy were within the expected ranges considering the manufacturing tolerances. At injection energy hysteresis effects make a significant contribution to the $b_{6}$ field component.

Following the satisfactory performance of the prototype, the MQTB design has been selected as the design to be used for the MQT correctors in the LHC, since it generates a greater field gradient and is expected to be the least expensive of the two designs to fabricate in series quantities.

\section{REFERENCES}

[1] M. Allitt et al., "Principles developed for the construction of the high performance, low-cost superconducting LHC corrector magnets," IEEE Trans. Appl. Superconduct..

[2] A. Ijspeert and J. Salminen, "Superconducting coil compression by scissor laminations," in EPAC-96, Sitges, Spain, June 1996.

[3] S. Russenschuck, "A computer program for the design of superconducting accelerator magnets," in Proc. ACES'95, Monterey, CA, Mar. 20-24, 1995. 